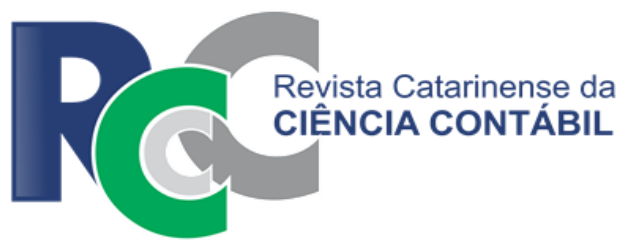

\title{
GASTOS COM VIGILÂNCIA EPIDEMIOLÓGICA NOS MUNICÍPIOS DE SANTA CATARINA
}

\author{
EPIDEMIOLOGICAL SURVEILLANCE COSTS IN THE CITIES OF SANTA \\ CATARINA
}

\author{
VALKYRIE VIEIRA FABRE \\ Universidade do Estado de Santa Catarina. Endereço: Rua Dr. Getúlio \\ Vargas, 2822 - CEAVI | Bela Vista | 89140-000| Ibirama/SC | Brasil. \\ (D) http://orcid.org/0000-0002-4664-1415 \\ valkyrie.fabre@udesc.br
}

\section{EMANUELE ENGELAGE}

Universidade Federal de Santa Catarina. Endereço: Campos Universitário CSE | Trindade | 88040-970| Florianópolis/SC | Brasil.

(D) http://orcid.org/0000-0002-9243-5209

manuengelage@hotmail.com

\section{LEONARDO FLACH}

Universidade Federal de Santa Catarina. Endereço: Campos Universitário CSE | Trindade | 88040-970| Florianópolis/SC | Brasil.

Dhttp://orcid.org/0000-0002-4316-0704

leonardo.flach@gmail.com

\author{
ALTAIR BORGERT \\ Universidade Federal de Santa Catarina. Endereço: Campos Universitário - \\ CSE | Trindade | 88040-970| Florianópolis/SC | Brasil. \\ Dhttp://orcid.org/0000-0002-0116-8089 \\ altair@borgert.com.br
}

\section{RESUMO}

A limitação de recursos e a priorização social têm feito com que a eficiência na saúde pública se torne pauta de diversos debates. Contudo, muitas vezes as escolhas dos governantes podem seguir prioridades individuais, conforme preconizado pela teoria das escolhas públicas, em especial na área epidêmica, que é fortemente influenciada pelo pânico e pela mídia. Diante disso, objetiva-se analisar a eficiência dos recursos públicos aplicados em vigilância epidemiológica, na mitigação de doenças como a Aids, a Dengue e a Gripe Influenza. Por meio de estatística descritiva, correlação e regressão de dados em painel, analisam-se 240 observações, em 60 municípios catarinenses, durante 4 anos. Os resultados indicam que os gastos explicam as ocorrências de Aids e de Gripe em sentido inverso ao sugerido pela literatura (quanto mais gastos, mais casos dessas doenças). Por outro lado, os casos das três doenças aumentam os gastos epidemiológicos. Portanto, as escolhas dos gestores parecem se direcionar mais para o tratamento do que para a prevenção

Resumo preliminar deste artigo foi apresentado no $54^{\circ}$ Congresso da Sociedade Brasileira de Medicina Tropical - Medtrop 2018. Recebeu a menção honrosa do Prêmio Chico Ribeiro em 2018, no Congresso Internacional de Contabilidade, Custos e Qualidade do Gastos Público.

Submissão em 02/12/2019. Revisão em 17/03/2020. Aceito em 08/04/2020. Publicado em 25/05/2020. 
das doenças, o que contraria os preceitos do Sistema Único de Saúde, aumenta os riscos de epidemias e pode agravar a crise na saúde pública. As discussões demonstram possíveis fatores ocasionais, como a falta de temporalidade na alimentação do sistema e distorções quanto ao local de incidência dos casos, o que também pode levar o gestor à tomada de decisão equivocada ou divergente daquela esperada por seus eleitores.

Palavras-chave: Saúde pública. Epidemias. Controle de custos. Eficiência. Teoria da escolha pública.

\begin{abstract}
Limitation of resources and social prioritization have led public health care to be guided by several debates. However, governments' choices can often follow individual priorities, as advocated by the public choice theory, especially in the epidemic area, which is strongly influenced by panic and the media. Therefore, the objective here is to analyze the efficiency of public resources applied in epidemiological surveillance in the mitigation of diseases such as AIDS, Dengue fever and Influenza. By means of descriptive statistics, correlation and panel data regression, 240 observations are analyzed in 60 municipalities in the Brazilian state of Santa Catarina for four years. The results indicate that the expenditures explain the occurrences of AIDS and Influenza in the opposite direction to that suggested by the literature (the more expenditures, the more cases of these diseases). On the other hand, cases of the three diseases increase epidemiological costs. Therefore, public administrators' choices seem to be directed more towards treatment than towards disease prevention, which goes against the Brazilian Unified Health Care System's precepts, increases the risks of epidemics and can aggravate the crisis in public health care. The discussions demonstrate possible occasional factors such as the lack of temporality in feeding the system and distortions regarding the place of incidence of the cases, which can also lead public administrators to make wrong decisions or diverge from those expected by their voters.
\end{abstract}

Keywords: Public health. Epidemics. Costs control. Efficiency. Theory of Public Choice.

\title{
1 INTRODUÇÃO
}

A saúde pública envolve uma grande área de atuação, sendo que a diversidade e a complexidade de suas atividades exigem altos investimentos. Zhu e Wang (2011) afirmam que a maioria dos países desenvolvidos gasta mais de $10 \%$ de seu orçamento governamental em cuidados com saúde, enquanto que o gasto de países em desenvolvimento gira em torno de $6 \%$. No Brasil, de acordo com o balanço anual consolidado de 2017, o percentual aplicado em saúde foi de $8,6 \%$ em relação ao total de gastos públicos (Secretaria do Tesouro Nacional [STN], 2017).

A necessidade de investimentos públicos em saúde se intensifica pelo fato de se tratar de um direito social estabelecido pela Constituição da República Federativa do Brasil (Constituição da República Federativa do Brasil [CRFB], 1988), a qual estabelece que é de competência dos governos (municipal, estadual e federal) zelar, proteger e defender a saúde da população. Liu e Mills (2002) corroboram essa ideia ao argumentar que o governo não deve se afastar da saúde como agente financiador e fiscalizador de ações públicas preventivas, e ressaltam que isso depende, também, de escolhas políticas, enquanto Holmes e Sunstein (2000) afirmam que o direito à saúde não pode ser protegido ou aplicado sem recursos públicos; portanto, os direitos geram gastos. Ainda, de acordo com Dih-Ling, Cheng-Chieh, Yun-Ru e Szu-Chieh (2018), o conhecimento dos gastos, juntamente com a determinação e caracterização das doenças, são fundamentais para subsidiar decisões que promovam a estabilidade financeira de hospitais e dos sistemas de saúde, além de embasar as diferentes formas de alocação dos recursos. 
Neste sentido, estudos da Malásia e da China apontam que, além de conhecer os gastos gerais com saúde pública, é necessário compreender a sua eficiência em atividades específicas (Worrall, Rietveld \& Delacollete, 2004; Liu \& Mills, 2002). Esses autores relatam a dificuldade na coleta de informações da saúde por área de atuação e afirmam que o seu conhecimento respalda decisões que precisam ser desassociadas, devido às características específicas de cada área. Além disso, Lapsley e Wright (2004) ressaltam as dificuldades dos pesquisadores em identificar o funcionamento contábil de cada governo, uma vez que a estrutura contábil não é padronizada internacionalmente, o que compromete a comparabilidade e a generalização dos estudos na área.

Segundo Morais, Vicente e Souza (2016), a classificação sintética utilizada pela Organização para Cooperação e Desenvolvimento Econômico (OCDE), com base nas Normas Internacionais de Contabilidade do Setor Público (IPSAS 22), é a mais utilizada na contabilidade mundial. No Brasil, a classificação é determinada pela Portaria n. 42, de 14 de abril de 1999 (STN, 2017), que estabelece como subfunções da saúde: atenção básica; assistência hospitalar e ambulatorial; suporte profilático e terapêutico; vigilância sanitária; vigilância epidemiológica; e alimentação e nutrição. Conforme Barbosa (2013), o campo das vigilâncias se refere às atividades necessárias para promover e fiscalizar a saúde dos cidadãos, e merece destaque na saúde coletiva devido à alta externalidade e à necessidade de ação rápida e abrangente, que demanda alta coordenação. Neste sentido, os gastos públicos com vigilâncias são classificados como epidemiológicos ou como sanitários (STN, 2017), cujas ações públicas são consideradas como de prevenção e mitigação, principalmente aquelas relacionadas à vigilância epidemiológica.

A Lei n. 8.080, de 19 de setembro de 1990, conhecida como Lei do SUS, define a subfunção "vigilância epidemiológica" como o conjunto de ações que proporcionam o conhecimento, a detecção e a prevenção de qualquer mudança que possa afetar a saúde individual ou coletiva, com finalidade de indicar e adotar as medidas de prevenção e controle das doenças ou agravos (Lei n. 8.080, 1990). Para Worrall et al. (2004), a saúde pública carece de pesquisas multidisciplinares sobre gastos eficientes, em especial na área epidêmica, em que as escolhas públicas muitas vezes são conduzidas pelo pânico e influenciadas fortemente pela mídia. Corroboram essa afirmação Lins, Ciríaco e Anjos (2019), que analisaram o impacto do financiamento federal no controle da Dengue no Brasil e afirmam que ainda há poucos estudos que buscam uma relação causal entre a destinação de verbas públicas e os casos notificados da doença, o que impossibilita inferências para suporte às decisões dos gestores públicos.

Além dessa limitação, existem vários fatores que podem dificultar a tomada de decisão na área de saúde pública. Segundo a teoria da escolha pública (TEP), de Buchanan e Tullock (1962), muitas vezes os gestores fazem escolhas que seguem prioridades individuais, ou de seu grupo, em detrimento de escolhas direcionadas pelo coletivo que os elegeu. Em geral, essas decisões não condizem com a busca pela eficiência dos recursos públicos de saúde nem com a erradicação das doenças e, assim, podem diminuir o bem-estar coletivo (Preaud et al., 2014).

Em função do exposto e considerando-se a necessidade de evitar desperdícios e maximizar resultados - principalmente para a prevenção de doenças, mas, também, para que não sejam negados tratamentos por falta de recursos (Dermindo, 2019) -, este estudo analisará a eficiência de recursos públicos aplicados em vigilância epidemiológica, na mitigação de doenças como a Aids, a Dengue e a Gripe Influenza. Assim, por meio de estatística multivariada, na qual relacionam-se os gastos públicos da área com os efetivos registros dessas doenças, além de variáveis populacionais e territoriais, analisam-se dados de municípios de Santa Catarina.

Em geral, as pesquisas identificadas sobre vigilância epidemiológica abordam o controle epidêmico, o desenvolvimento de medicamentos e os meios de disseminação de doenças. Portanto, esta pesquisa traz uma visão diferenciada, ao abordar o viés financeiro por meio da análise de eficiência dos gastos públicos. Também diferencia-se de estudos como o de Lins et al. (2019), na medida em que trata dos gastos epidêmicos relacionados a diversas doenças, enquanto aquele limita-se à análise de recursos destinados a um único programa, com ações de controle apenas da Dengue. Apesar de pouco explorada, a relação entre gastos e doenças epidêmicas tem ganhado 
mais destaque em 2020, com a pandemia de Covid-19, visto que, em situações como esta, apesar do alto custo para os governos, este pode ser inferior ao custo social e macroeconômico (McKibbin \& Roshen, 2020).

A ligação entre saúde da população e aspectos econômicos, sem se restringir a questões epidêmicas, já foi objeto de estudos anteriores em diversos países (Bousmaha, Ventelou \& AbuZaineh, 2016; Araújo, Bezerra, Amâncio, Passos \& Carneiro, 2017; Ensor, Chhun, Kimsun, McPake \& Edoka, 2017), assim como os gastos governamentais com saúde (Liu \& Mills, 2002; Zhu \& Wang, 2011; Pan \& Liu, 2012; Halici-Tülüce, Dogan \& Dumrul, 2016; Mladenovi, Milevancevi, Mladenovi, Marjanovi \& Petkovi, 2016; Younsi, Chakroun \& Nafluma, 2016; Bein, Unlucan, Olowu \& Kalifa, 2017; Costa \& Gartner, 2017). Porém, não há um consenso quanto à melhor abordagem para aferir o desempenho de sistemas de saúde, já que as escolhas públicas e as características de cada amostra levam a resultados diferentes (Albuquerque et al., 2019). O que é semelhante entre esses estudos é a utilização de gastos gerais com saúde, uma vez que extrair dados específicos por área nem sempre é possível. Neste quesito, o Brasil tem um potencial ainda pouco explorado, haja vista que a contabilidade pública é mais detalhada, o que permite análises por subfunção.

Cabe destacar que a maioria dos estudos identificados como base para esta pesquisa utiliza a classificação contábil padrão da OCDE, que insere em uma conta sintética todos os gastos com saúde pública. Porém, compreender os reflexos dos gastos alocados em áreas específicas pode trazer insights importantes na busca por maior eficiência. Assim, uma das contribuições do presente estudo é direcionar as análises aos gastos apenas com vigilância epidemiológica, uma área importante dentro da saúde coletiva, no sentido de produzir conhecimentos para orientar a tomada de decisão, inclusive na formulação de políticas, organização de serviços e programas de governo (Barreto \& Souza, 2015).

\section{FUNDAMENTAÇÃO TEÓRICA}

A TEP surgiu com base no clássico estudo de Buchanan e Tullock (1962), cuja lente teórica foca na escolha incerta quanto ao futuro resultado do bem-estar social, pois decisores possuem auto interesse direto e deixam de lado questões políticas ordinárias. Para Fabre, Scheeffer, Dallabona e Kroetz (2018), a TEP explica o interesse particular dos gestores em sobreposição ao interesse da sociedade quando o assunto são as políticas públicas do governo.

Contudo, para compreender as reais motivações por trás das decisões e escolhas dos gestores, primeiramente é necessário conhecer comportamentos específicos, especialmente aqueles que se relacionam à aplicação de recursos. Na área da saúde, pesquisas internacionais recentes sobre gastos públicos (Bousmaha et al., 2016, Ensor et al., 2017) relacionam a saúde da população com o desenvolvimento econômico, ao mesmo tempo em que buscam caracterizar tais gastos.

Bousmaha et al. (2016) estudaram os gastos públicos em países do Oriente Médio e Norte da África e verificaram que instituições públicas com melhor estrutura conseguem diminuir gastos. Contudo, nem sempre a diminuição dos gastos beneficia a todos. A experiência do Camboja, descrita por Ensor et al. (2017), revela benefícios diferentes para classes sociais distintas. No país, há uma política governamental que oferece acesso à saúde para todos e, em alguns casos, há fornecimento de voucher de coparticipação, ou seja, uma parte é paga pelo governo e a outra pelo cidadão. Para os autores, essa política beneficia as classes mais ricas, que ocupam a maior parte dos serviços públicos, e desfavorece as mais pobres, pois aumenta o comprometimento de sua remuneração com os gastos individuais em saúde.

Ainda, quanto aos gastos públicos em saúde, Zhu e Wang (2011) afirmam que não há um padrão desses recursos ou uma associação com o desempenho econômico dos países. Isso porque, ao analisar o percentual destinado para ações de saúde, do total de recursos disponíveis em cada país, os autores identificaram resultados consideravelmente distintos, dentre os quais citam-se: 
Estados Unidos 21\%; Austrália 18,2\%; República Checa 14,5\%; Alemanha 13,6\%; Rússia 12,7\%; Suazilândia 11,4\%; Romênia 10,7\%; Polônia 10,3\%; Bolívia 9,6\%; Irã 6\%; Singapura 5,8\% e China 4\%. Apesar dessas diferenças, segundo Younsi et al. (2016), que analisaram 167 países de baixa, média e alta renda, algo comum a todos eles é que o gasto total com saúde tem aumentado nas últimas décadas.

Mesmo com essa elevação, e independentemente do desenvolvimento econômico, a Organização Mundial da Saúde (OMS) sugere que os países ou regiões devem tomar medidas para aumentar a eficiência dos serviços de saúde a fim de maximizar os resultados obtidos com um dado nível de recursos, ou minimizar os recursos necessários para obter determinado resultado (Dermindo, 2019). Nessa direção, de acordo com Mladenovi et al. (2016), a maioria dos estudos confirmam a relação positiva entre gastos públicos e melhorias na saúde. Para os autores, que analisaram países da União Europeia, a adição de recursos destinados a cuidados médicos e de saúde pública (equivalente à atenção básica brasileira) implica melhoria do estado de saúde da população e forma a melhor combinação para a previsão da taxa de crescimento do PIB.

No Brasil, o efeito da alocação de recursos em saúde e educação foi analisado por Costa e Gartner (2017); os quais concluíram que maiores gastos em algumas subfunções da saúde (atenção básica, assistência hospitalar, suporte profilático e ambulatorial) e na educação infantil produzem melhorias no bem-estar dos trabalhadores e reflexos positivos no aumento da renda nos estados pesquisados. Já ao analisar a eficiência de recursos aplicados em ações específicas de saúde, Lins et al. (2019) constataram que os gastos públicos federais destinados ao programa de redução da Dengue têm surtido efeito positivo, com redução dos casos da doença. Os autores analisaram 4.956 municípios brasileiros com menos de 50 mil habitantes, de 2002 a 2015 e, apesar da melhora identificada, haja vista que desde 2002 há esforços para diminuir o número de casos por meio da prevenção e da redução na proliferação do mosquito, ainda há forte heterogeneidade na resposta entre as regiões do país. Isso se deve, principalmente, pelas diferenças nos níveis de desenvolvimento econômico e social.

Albuquerque et al. (2019) também versam sobre as diferenças entre os níveis de desenvolvimento, ao avaliar o desempenho da regionalização da vigilância em saúde no Brasil. Os autores estabelecem três aspectos de avaliação: política, estrutura e organização, para os quais há diferentes atributos. A metodologia adotada não visa traçar uma relação direta desses aspectos com dados financeiros, apesar de estarem intrinsicamente vinculados. Mesmo assim, os achados ganham relevância pois revelam, sob o ponto de vista dos atores-chave da vigilância que responderam ao questionário, que há insuficiente disponibilidade de recursos físicos, humanos e financeiros, o que dificulta o avanço da política de regionalização e o alcance dos macro objetivos. Além disso, os referidos autores concluem que, quanto maior o nível de desenvolvimento econômico e da oferta de serviços, melhor o desempenho da regionalização da vigilância em saúde.

Da mesma forma, os impactos dos gastos na saúde da população não seguem um padrão mundial. Ao analisar 44 países (de alta e de baixa renda), num espaço temporal de 17 anos, HaliciTülüce et al. (2016) concluíram que os gastos em saúde pública e privada não apresentam retorno no crescimento econômico em curto prazo. Entretanto, no longo prazo a saúde pública exerce forte impacto positivo no crescimento econômico e no bem-estar da população, enquanto a saúde privada apresenta impacto negativo. Contrariando tais resultados, por meio de um estudo sobre os efeitos das políticas de saúde públicas e privadas na África, Bein et al. (2017) afirmam que existe uma forte associação entre o aumento dos gastos públicos e o aumento imediato na saúde da população, enquanto que os gastos de instituições privadas possuem resultado na população em médio prazo.

Na China, Pan e Liu (2012) relacionaram a qualidade da saúde pública com o PIB e concluíram que os gastos do governo não influenciam naquele indicador nem na saúde da população, o que torna-os gastos ineficientes. Contudo, para Liu e Mills (2002), os gastos com saúde pública surtem efeitos positivos no combate a certas doenças, ao analisar a reforma de saúde ocorrida na China, caracterizada pela redução no orçamento público e a introdução de cobranças 
pela prestação dos serviços. Os autores concluíram que, apesar de trazer incentivos econômicos às instituições privadas e melhorar a produtividade das instituições públicas, os serviços preventivos foram drasticamente reduzidos pela cobrança de tarifas, o que gerou um aumento considerável de doenças epidemiológicas. Contudo, em estudo sobre o custo da pandemia do Covid-19, McKibbin e Roshen (2020) afirmam que prevenir epidemias parece ser a saída mais eficiente para evitar maiores proporções, que resultem em prejuízo à vida e à macroeconomia.

Ainda quanto às doenças epidêmicas, Dih-Ling et al. (2018) trazem uma concepção diferenciada ao inverter a lógica de análise dos estudos supramencionados. Isso porque os autores analisaram os efeitos econômicos da Dengue em Taiwan, no período de 1998 a 2014; ou seja, ao invés de verificar como a aplicação de recursos impacta nos casos dessa doença (eficiência), verificaram como os casos de Dengue impactam no comportamento dos gastos, ao comparar anos epidêmicos e não epidêmicos (consequências). Para caracterizar tais gastos, os autores os dividiram em diretos (custos médicos) e indiretos (diminuição da produtividade e remuneração do cuidador), cujos resultados indicaram que os gastos foram 12,3 vezes mais altos em anos epidêmicos. Além disso, constataram que do total de despesas diretas desse período, 86,9\% vinculam-se à hospitalização, 7,77\% com emergência, 6,1\% com ambulatório e $0,03 \%$ com medicamentos. Já para os gastos indiretos, destacam-se a perda de produtividade devido a óbito $(70,76 \%)$.

Araújo et al. (2017) também reconhecem os amplos efeitos das doenças epidêmicas. Segundo os autores, as epidemias precisam ser controladas, pois causam perda de saúde temporária ou definitiva, enfraquecem postos de trabalho e prejudicam não só a saúde, mas a própria economia da região atingida. Segundo Preaud et al. (2014), se os países seguissem a orientação da OMS e do Centro Europeu de Controle de Doenças (CECD), e ampliassem a vacinação e as ações preventivas, poderiam reduzir os casos dessas doenças em todo o mundo, aumentar a eficiência dos recursos públicos de saúde, preservar a saúde das pessoas, impedir a perda de produtividade e estimular o crescimento econômico.

Neste sentido, Naseem, Rashid e Kureshic (2014) destacam que a informação desempenha papel fundamental para uma vigilância eficaz, principalmente no combate às epidemias, ao observarem os efeitos dos sistemas eletrônicos de informação da saúde (e-saúde) na relação médico-paciente e a capacidade dos profissionais de saúde em relação ao uso de tecnologia de informação, no Paquistão. Para os autores, apesar de a tecnologia e o e-saúde serem importantes para controlar doenças epidemiológicas, atualmente há escassez de profissionais qualificados e de ferramentas tecnológicas na rede pública do país.

Diante do exposto, verifica-se que o papel dos governos na promoção da saúde pública pelo poder de disseminação das informações (Naseem et al., 2014), treinamento de pessoal, disponibilização de infraestrutura, políticas voltadas à vigilância e campanhas preventivas e de combate às doenças epidêmicas (Liu \& Mills, 2002), possui como resultado maior eficiência dos serviços no curto e médio prazos (Bein et al., 2017), com melhoria da saúde da população. A consequência disso é a relação positiva entre gastos com saúde pública e o desenvolvimento econômico das nações (Mladenovi et al., 2016, Costa \& Gartner, 2017, Bein et al., 2017).

\section{PROCEDIMENTOS METODOLÓGICOS}

O presente estudo caracteriza-se como empírico, com abordagem predominantemente quantitativa, objetivo descritivo e com procedimentos de levantamento de dados, uma vez que analisa a eficiência dos gastos com vigilância epidemiológica na mitigação de doenças específicas. Cabe destacar que, no Brasil, várias epidemias atingem a população anualmente. No entanto, por se tratar de um país de dimensões continentais, com climas diversos e características distintas entre as regiões, optou-se por analisar apenas quatro variáveis de doenças epidêmicas, quais sejam: Gripe Influenza, Dengue casos, Dengue focos e Aids em adultos. Essa delimitação ocorre por se tratar das doenças mais incidentes em Santa Catarina, região selecionada para esta pesquisa, na 
medida em que os dados necessários se apresentam disponíveis pela Diretoria de Vigilância Epidemiológica de Santa Catarina (Diretoria de Vigilância Epidemiológica [Dive], 2017).

Selecionou-se uma amostra aleatória de 60 municípios, dentre os 295 catarinenses. Inicialmente, realizou-se a estratificação por faixas habitacionais: grandes, médios e pequenos municípios. Em seguida, por meio do software Microsoft Excel®, selecionou-se 20 municípios pertencentes a cada grupo (faixa), cuja relação se apresenta na Tabela 1.

\section{Tabela 1}

\section{Amostra da pesquisa}

\begin{tabular}{ll}
\hline Faixa habitacional & Municípios Selecionados \\
\hline $\begin{array}{l}\text { Grandes municípios } \\
\text { (50.001-600.000 }\end{array}$ & Joinville, Caçador, Blumenau, São José, Criciúma, Chapecó, Itajaí, Lages, Jaraguá do \\
habitantes) & Sul, Palhoça, Balneário Camboriú, São Bento do Sul, Concórdia, Camboriú, \\
\hline $\begin{array}{l}\text { Médios municípios } \\
\text { (10.001-50.000 }\end{array}$ & Videgantes, Rio do Sul, Araranguá, Indaial, Canoinhas e Itapema. \\
habitantes) & Schroeder, Taió, Jaguaruna, Garopaba, Guabiruba, São Joaquim, Urussanga, Xaxim, \\
\hline \multirow{2}{*}{$\begin{array}{l}\text { Pequenos municípios } \\
\text { (0-10.000 habitantes) }\end{array}$} & São João Batista, Braço do Norte, Timbó, Rio Negrinho e Ibirama. \\
\hline Fonte: Elaborada pelos autores , Bom Jesus do Oeste, São Bernardinho, Marema, Ouro Verde, Abdon \\
Sul, Luzerna, Caibi, Petrolândia, Água Doce, Ouro, Agrolândia e São José do Cerrito.
\end{tabular}

Dada a amostra, analisou-se como os gastos municipais totais, ou seja, os recursos públicos de fonte própria ou não, aplicados em vigilância epidemiológica, afetam o número de ocorrências de cada doença durante os anos de 2013 a 2016. A definição do lapso temporal ocorreu devido à TEP, que estabelece que os recursos públicos são destinados às ações escolhidas pelos gestores eleitos. Portanto, selecionou-se o período correspondente ao último mandato completo dos prefeitos. Já quanto ao número de ocorrências, apesar de as avaliações de desempenho da vigilância em saúde utilizarem diferentes abordagens metodológicas no Brasil (Albuquerque et al., 2019), optou-se por analisar o desempenho epidemiológico através do efetivo registro de casos, assim como Liu e Mills (2002) e Lins et al. (2019). A Tabela 2 apresenta a definição de todas as variáveis do estudo e as respectivas fontes de coleta de dados.

Tabela 2

\section{Variáveis e fontes pesquisadas}

\begin{tabular}{ll}
\hline \multicolumn{1}{c}{ Variáveis utilizadas } & \multicolumn{1}{c}{ Fonte de pesquisa } \\
\hline & Balanços públicos disponibilizados no Sistema de Coleta de \\
Gastos públicos municipais de saúde, & Dados Contábeis (SISTN) ou no Sistema de Informações \\
alocados na subfunção vigilância & Contábeis e Fiscais do Setor Público Brasileiro (Siconfi): \\
epidemiológica. & Relatório Resumido da Execução Orçamentária (RREO), \\
& Anexo 2, subfunção 305 - Vigilância epidemiológica. \\
\hline Casos de Gripe Influenza; Casos e focos de & $\begin{array}{l}\text { Diretoria de Vigilância Epidemiológica de Santa Catarina } \\
\text { Dengue; Casos de Aids em adultos. }\end{array}$ \\
\hline Número de habitantes; área territorial $\left(\mathrm{Km}^{2}\right)$. & Instituto Brasileiro de Geografia e Estatística (IBGE). \\
\hline
\end{tabular}
Fonte: Elaborada pelos autores (2019).

Após a coleta, os dados foram tabulados e organizados por meio do software Microsoft Excel ${ }^{\circledR}$. Ao todo, a base de dados resultou em 240 observações, que contemplam 60 municípios, durante 4 anos. Para a análise dos dados utilizou-se, primeiramente, a técnica de estatística descritiva para uma melhor compreensão das variáveis, pois, conforme sugerido por Dih-Ling et al. (2018), a caracterização da doença e de seus gastos é importante para identificar possibilidades de alocação de recursos. Também utilizou-se os métodos estatísticos multivariados de correlação de Pearson e regressão para dados em painel, similarmente ao trabalho de Younsi et al. (2016), 
sob a justificativa de que tais consideram tanto as características de cross-section, ou seja, as diferenças entre os indivíduos, quanto as diferenças de séries de tempo.

Para alcançar o objetivo proposto estabeleceu-se quatro modelos equacionais, com diferenças apenas quanto à variável dependente, ou seja, os casos das doenças: Gripe Influenza, Dengue e Aids em adultos, com destaque para a Dengue que é segregada em casos e focos. Essa segregação ocorre, pois, a vigilância epidemiológica desenvolve ações que proporcionam o conhecimento, a detecção e a prevenção de doenças (Dive, 2017) e, neste caso, o controle de focos é considerada uma ação preventiva (Lins et al., 2019). Assim, o modelo de casos de Dengue inclui o número de focos como uma variável explicativa, pois entende-se que quanto maior for esse número, maior o risco de contrair a doença (Araújo et al., 2017). Outra particularidade a ser destacada é sobre a Aids: por se tratar de uma doença que ainda não tem cura e, portanto, o número de casos é cumulativo (Dive, 2017), no presente estudo considera-se apenas os novos casos detectados em cada período, o que permite visualizar a evolução na propagação da doença.

A variável explicativa dos modelos é o montante de gastos alocados à subfunção vigilância epidemiológica, enquanto a população e a área territorial são variáveis de controle, que visam isolar as diferenças de tamanho entre os municípios para que os índices sejam parâmetros para todos os modelos. Por se tratar de uma regressão de dados em painel, realizou-se a análise do melhor modelo de estimação, com verificação dos pressupostos básicos: normalidade, homocedasticidade e inexistência de autocorreção e multicolineariedade (Gujarati, 2006).

Como limitações, destaca-se o fato de que não é possível separar os gastos públicos por tipo de doença. A fonte dos dados relativos aos gastos brasileiros apresenta a classificação normativa padrão, que é mais analítica que a internacional. Apesar disso, alguns gastos epidemiológicos podem ser contabilizados em outras subfunções, quando relacionados a outros procedimentos de saúde. Além disso, o período analisado não foi avaliado quanto à possibilidade de conter problemas epidêmicos remanescentes de anos anteriores.

\section{RESULTADOS}

Para verificar a evolução temporal dos dados, na Figura 1 demonstra-se graficamente a média de todos os municípios para cada ano, das variáveis "gastos" e "casos de doenças".

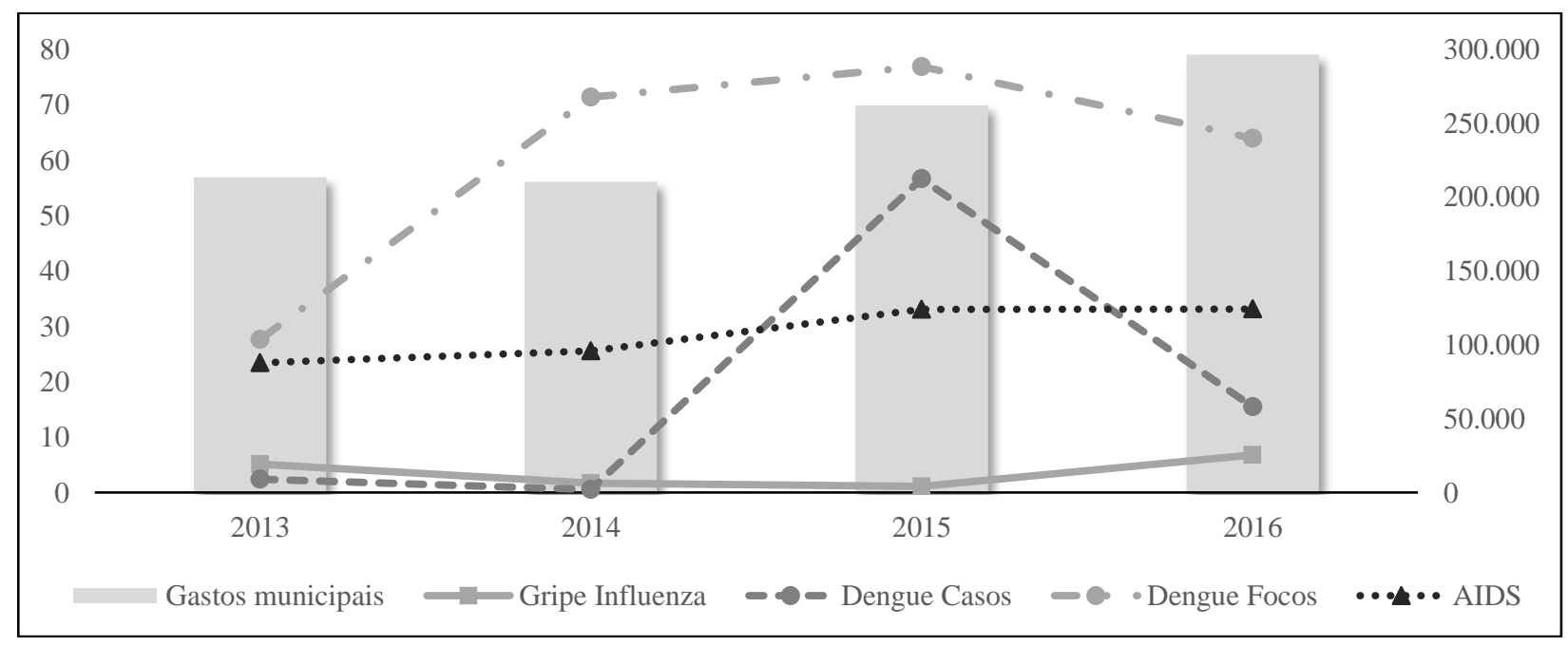

Figura 1. Evolução temporal de gastos e casos de doenças Fonte: Elaborada pelos autores com base nos dados da pesquisa (2019).

Quanto aos gastos municipais, pode-se constatar que após uma pequena redução entre os anos de 2013 e 2014, correspondente a 1,35\% (de $\mathrm{R} \$ 213.156,22$ para $\mathrm{R} \$ 210.287,36$ ), até o ano de 2016 há elevação da média de recursos destinados à vigilância epidemiológica nos 60 
municípios analisados ( $\mathrm{R} \$ 261.91626$ em 2015 e $\mathrm{R} \$ 296.336,96$ em 2016). Ou seja, em relação ao ano imediatamente anterior, em 2015 os gastos nessa área cresceram 24,55\%, e em 2016 mais 13,14\%. Esse fato corrobora as pesquisas de Younsi et al. (2016), de que os gastos totais com saúde têm aumentado ao longo do tempo.

Contudo, mesmo com esses investimentos, os casos de doenças também se elevaram nesse período. No ano de 2014 houve expressivo crescimento nos focos do mosquito da Dengue: em 2013 era um total de 1.658 focos nos 60 municípios analisados (média de 27,63 por município), enquanto em 2014 passou para 4.280 focos (média de 71,33 por município), mantendo-se praticamente estável nos anos seguintes. Isso fez com que os casos de Dengue aumentassem consideravelmente, passando de um total de 31 em 2014 (média de 2,37 por município) para 3.397 casos em 2015 (média de 56,61 por município), o que caracterizou uma epidemia dessa doença no país (Araújo et al., 2017). O acréscimo nos casos de Dengue nesse período não é uma exclusividade dos municípios catarinenses. Os estudos de Araújo et al. (2017) e de Lins et al. (2019), mesmo com amostras distintas, demonstram que todas as regiões e estados brasileiros tiveram um acréscimo nos casos da doença. Lins et al. (2019) destacam ainda que, segundo o Ministério da Saúde, no ano de 2015, das cidades com situação epidêmica (acima de 300 casos notificados para cada grupo de 100 mil habitantes), 87\% são pequenos municípios com menos de 50 mil habitantes. Já a Gripe Influenza manteve-se estável ao longo dos quatro anos analisados, com maior incidência em 2016, quando ocorreram 407 casos (média de 6,78 por município). Para a Aids em adultos, as variações também são menores, mas em sentido crescente ao longo dos quatro anos. Em 2013, por exemplo, foram detectados 1.404 casos, enquanto que em 2016 foram 1.986 casos.

Para dar completude a essas análises, apresenta-se na Tabela 3 as estatísticas descritivas das variáveis de todos os municípios em todos os anos.

Tabela 3

\section{Estatística descritiva}

\begin{tabular}{lrrrrrrr}
\hline & Gasto & Influenza & Dengue casos & Dengue focos & Aids & População & Área \\
\hline Média & $245.424,20$ & 3,68 & 18,75 & 59,94 & 28,78 & $62.000,08$ & 407,75 \\
Erro padrão & $29.432,09$ & 0,58 & 13,42 & 14,99 & 3,61 & $6.267,64$ & 29,48 \\
Mínimo & 0 & 0 & 0 & 0 & 0 & 1.254 & 46,24 \\
Quartil inferior & 0 & 0 & 0 & 0 & 0 & 5.964 & 160,9 \\
Mediana & $28.289,05$ & 0 & 0 & 1 & 1 & $21.873,50$ & 251,11 \\
Quartil superior & $275.346,59$ & 4 & 1 & 13 & 28,5 & $69.464,25$ & 447,76 \\
Máximo & $2.330 .115,86$ & 70 & 3.128 & 2.686 & 278 & 569.645 & $2.631,5$ \\
Desvio padrão & $455.959,91$ & 8,98 & 207,92 & 232,27 & 55,89 & $97.097,94$ & 456,78 \\
Curtose & 6,61 & 25,88 & 212,31 & 72,21 & 6,02 & 11,57 & 9,19 \\
Assimetria & 2,56 & 4,64 & 14,32 & 7,47 & 2,51 & 3,07 & 2,79 \\
Coef. de variação & 1,86 & 2,44 & 11,09 & 3,87 & 1,94 & 1,57 & 1,12 \\
\hline
\end{tabular}

Fonte: Elaborada pelos autores com base nos dados da pesquisa (2019).

De acordo com a Tabela 3, o gasto médio anual, por município, destinado à vigilância epidemiológica, é de R\$ 245.424,20. Em determinados anos, existem municípios que não destinaram recurso para esta subfunção. Por outro lado, o município que mais investiu foi Criciúma, em 2016, com o total de $\mathrm{R} \$ 2.330 .115,86$. Cabe destacar que entre os diversos fatores de risco que podem influenciar a necessidade de gastos com vigilância epidemiológica, tem-se o nível populacional. No caso de Criciúma, um município de grande porte, no ano em que apresentou o maior gasto (2016), a população era de 209.153 habitantes, valor acima do quartil superior.

Ainda quanto à população, a média da amostra é de 62 mil indivíduos por município, com variação entre 1.254 (Jaguaruna) e 569.645 (Joinville). Devido a essa disparidade, há diferença significativa entre a média e a mediana, o que é esperado, já que a amostra é estratificada por faixas habitacionais. Da mesma forma, existem municípios maiores e menores em termos de área, ou seja, extensão territorial. A média territorial da amostra é de $407,75 \mathrm{~km}^{2}$, sendo que o munícipio 
de Balneário Camboriú é o menor, com apenas 46,24 km², e Lages é o maior, com 2.631,50 km². A estratificação da amostra, ao considerar 20 municípios de cada faixa habitacional, também justifica as diferenças entre a média e a mediana das demais variáveis em estudo, pois em todos os casos os valores extremos inferior e superior são consideravelmente dispersos.

Quanto às doenças, a maior média de ocorrência é de Aids (28,78 casos). Isso porque, os focos do mosquito transmissor da Dengue, que tem média de 59,94, não representam doença efetiva. Apesar de a Aids ter a maior média, ao considerar todos os municípios em todos os anos, a doença com maior ocorrência é a Dengue, pois no ano de 2015, quando houve uma epidemia no Brasil, o município de Itajaí registrou 3.128 casos, o maior registro de todas as doenças em todos os anos e municípios. Contudo, devido ao fato de diversos municípios, em diferentes anos, não registrarem qualquer caso de Dengue, o valor médio dessa doença é reduzido. Embora Itajaí apresente o maior número de casos de Dengue, não é o que possuiu maior número de focos, porque em 2014 o município de Chapecó apurou 2.686 focos do mosquito transmissor, sendo que nesse mesmo período ocorreram apenas três casos da doença no município. Contudo, nos anos posteriores, Chapecó teve um aumento considerável de casos, pois em 2015 notificou 45 pessoas infectadas e, em 2016, 785. Isso demonstra que pode ocorrer um delay entre o período de proliferação do Aedes aegypti (vetor primário da transmissão) e o efetivo contágio. Além disso, a existência do mosquito não representa, necessariamente, a ocorrência da doença, pois o vírus é transmitido pela picada da fêmea apenas quando esta já está infectada (Araújo et al., 2017).

Diante desses achados, é importante, também, analisar a relação dessas variáveis, ou seja, dos focos e dos casos de Dengue. Para a compreensão da relação entre todas variáveis, apresentase na Tabela 4 os resultados da correlação de Pearson.

Tabela 4

Teste de correlação de Pearson

\begin{tabular}{llllllll}
\hline & Gasto & Influenza & Dengue casos & Dengue focos & AIDS & População & Área \\
\hline Gasto & 1 & & & & & & \\
Influenza & $0.4268^{*}$ & 1 & & & & & \\
Dengue casos & -0.0331 & 0.0413 & 1 & & & & \\
Dengue focos & 0.0601 & 0.0895 & $0.273^{*}$ & 1 & & & \\
Aids & $0.5607^{*}$ & $0.583^{*}$ & $0.201^{*}$ & $0.3386^{*}$ & 1 & & \\
População & $0.4887^{*}$ & $0.633^{*}$ & $0.1377^{*}$ & $0.2967^{*}$ & $0.8868^{*}$ & 1 & \\
Área & 0.0305 & $0.1681^{*}$ & -0.0082 & 0.0069 & $0.1558^{* *}$ & $0.2794^{*}$ & 1 \\
\hline
\end{tabular}

Nota. *A correlação é significativa no nível de $1 \%$.

** A correlação é significativa no nível de 5\%.

Fonte: Elaborada pelos autores com base nos dados da pesquisa (2019).

Com base na Tabela 4 é possível inferir que a maioria das variáveis está correlacionada ao nível de confiança de $99 \%$. Dentre as relações significativas, os gastos apresentam correlação positiva com a Gripe Influenza, com a Aids e com a população. Contudo, esperava-se que a relação entre gastos e essas doenças fosse inversa (negativa), ou seja, uma destinação maior de recursos poderia prevenir as doenças e ocasionar redução de sua ocorrência, o que não se confirmou. Essa expectativa foi criada em função do viés preventivo dado à subfunção de vigilância epidemiológica (Dive, 2017). Além disso, os estudos de Liu e Mills (2002), Bein et al. (2017) e Lins et al. (2019) evidenciaram que maiores gastos públicos surtem efeitos positivos no combate a certas doenças, com redução de casos.

Nota-se também que as variáveis de casos de Dengue e de focos do mosquito transmissor da doença são correlacionadas positiva e significativamente, o que confirma que mais focos podem aumentar o risco de contaminação, diferentemente do que foi verificado no município de Chapecó em 2014. Também destaca-se que a Aids é correlacionada positiva e significativamente com todas as doenças.

Por fim, conforme já mencionado, percebe-se que o tamanho da população se correlaciona positiva e significativamente com todas as variáveis, o que indica que municípios mais populosos 
possuem maiores gastos com vigilância epidemiológica, casos de doenças e focos do mosquito transmissor da Dengue. Numa análise complementar, utilizando-se o gasto per capita em vez do gasto total, verifica-se que o coeficiente passa a representar uma relação inversa entre gastos e população, porém não apresenta significância estatística para permitir qualquer inferência sustentável. Cabe destacar que, no caso de doenças epidêmicas, o uso de um valor per capita pode enviesar as análises, uma vez que há diferentes grupos de risco, que fazem com que maiores gastos se destinem a uma parcela reduzida da população, enquanto poucos recursos são destinados a um grupo maior de pessoas. Assim, o uso de uma média entre toda a população não condiz com a realidade e, por isso, optou-se por manter as análises com o gasto total e utilizou-se o número de habitantes (população) e a área total como variáveis de controle. Desta forma além do tamanho, pode-se captar também a densidade demográfica, já que se considera o nível populacional e a área, que são as variáveis utilizadas nesse indicador.

Isto posto, parte-se para a análise da regressão de dados em painel. Primeiramente, destacase que todos os modelos apresentam heterocedasticidade, autocorrelação e não normalidade dos resíduos. Diante disso, e ao considerar os testes do melhor modelo de estimação, a Tabela 5 apresenta os resultados da regressão pelo modelo Pooled Ordinary Least Square (Pols) com errospadrão robustos clusterizados, na medida em que este se apresenta como o mais adequado.

Tabela 5

\section{Resultados das regressões pelo modelo Pols com erros-padrão robustos clusterizados}

\begin{tabular}{|c|c|c|c|c|c|c|c|c|}
\hline & \multicolumn{2}{|c|}{ Gripe Influenza } & \multicolumn{2}{|c|}{ Dengue casos } & \multicolumn{2}{|c|}{ Dengue focos } & \multicolumn{2}{|c|}{ Aids } \\
\hline $\mathrm{N}^{\circ}$ de observações & & 240 & & 240 & & 240 & & 240 \\
\hline Prob > F & & 0 & & 0,7848 & & 0,0844 & & 0 \\
\hline R-Squared & & 0,4189 & & 0,088 & & 0,106 & & 0.8138 \\
\hline & $\mathbf{P}>\mathbf{t}$ & Coef. & $P>t$ & Coef. & $\mathbf{P}>\mathbf{t}$ & Coef. & $\mathbf{P}>t$. & Coef. \\
\hline Gasto & 0,003 & 0,00000306 & 0,241 & $-0,0000507$ & 0,462 & $-0,0000636$ & 0,021 & 0,0000191 \\
\hline Dengue focos & - & _ & 0,353 & 0,2153793 & - & - & - & - \\
\hline População & 0,000 & 0,0000513 & 0,326 & 0,0002872 & 0,123 & 0,0009204 & 0,000 & 0,0004797 \\
\hline Área & 0,783 & 0,0001642 & 0,289 & $-0,0198338$ & 0,015 & $-0,0492266$ & 0,057 & $-0,0100149$ \\
\hline _cons & 0,191 & $-0,3185854$ & 0,343 & 8,740591 & 0,021 & 38,56407 & 0,474 & $-1,568858$ \\
\hline
\end{tabular}

Fonte: Elaborada pelos autores com base nos dados da pesquisa (2019).

Os resultados indicam que os modelos de Gripe Influenza e AIDS são significativos ao nível de confiança estabelecido neste estudo (90\%) e as variáveis independentes conjuntas explicam, respectivamente, $41,89 \%$ e $81,38 \%$ dos casos das doenças. Para a Gripe Influenza as variáveis "gasto" e "população" possuem significância estatística. Já para a Aids em adultos, todas as variáveis são significativas, ou seja, o gasto, a população e a área. Dado o coeficiente positivo da variável "gasto" em ambos os modelos, assim como verificado na correlação de Pearson e ao contrário do esperado pela literatura em que os investimentos em saúde previnem doenças e, consequentemente, diminuem sua ocorrência (Liu \& Mills, 2002, Bein et al., 2017, Lins et al., 2019), verifica-se que, estatisticamente, a aplicação de recursos em vigilância epidemiológica eleva o número de casos dessas doenças. Diante disso, pode-se afirmar que os gastos alocados a essa subfunção impactam os casos de Gripe Influenza e Aids, mesmo que possuam um coeficiente consideravelmente baixo (para cada $\mathrm{R} \$ 1,00$ tem-se 0,00000306 de acréscimo nos casos de Gripe e 0,0000191 de acréscimo nos casos de Aids).

A explicação para tal comportamento respalda-se em motivos diversos. Segundo Santos, Tayra, Silva, Buchalla e Laurenti (2002), o principal problema no caso da Aids é o atraso na chegada dos dados para inclusão no sistema de informação de epidemias. Essa dificuldade devese à facilidade de acesso aos medicamentos e às inúmeras ferramentas da medicina, que retardam os sintomas e muitas vezes os diagnósticos, fazendo com que os casos entrem cada vez mais tarde no sistema e inviabilizem um planejamento sobre a sua real situação. Portanto, as escolhas dos gestores municipais quanto aos recursos destinados às ações de prevenção dessa doença podem 
ser influenciadas, também, pela limitação informacional, e não apenas por interesses pessoais, como previsto na TEP.

Outra questão a ser levantada é que o diagnóstico de Aids, muitas vezes, é registrado nos grandes centros e não nos municípios de origem do caso, o que acaba por distorcer a informação por município. Um exemplo é Itajaí, que na década de 1990 foi citado como o município que mais tinha casos de Aids no estado de Santa Catarina. Inclusive, em 1998, caracterizou-se como o município com maior taxa de incidência do Brasil: 142,3 casos para cada 100 mil habitantes (Diretoria de Vigilância Epidemiológica [Dive], 2006). Ocorre que o Hospital Regional, com sede nessa cidade, era um dos poucos habilitados para a realização do exame na época. Assim, pessoas de vários municípios, e até estrangeiros (já que é uma cidade portuária), eram diagnosticadas como soropositivas de Itajaí.

Quanto à Gripe Influenza, destaca-se que em 2009 a OMS comunicou a todos os países uma emergência em saúde pública de importância internacional, causada pela infecção do vírus influenza tipo A, conhecido por $\mathrm{H}_{1} \mathrm{~N}_{1}$ (Temporão, 2009). Desde então, diante de aumentos nos casos dessa doença, o governo brasileiro passou a disponibilizar a vacina gratuitamente para alguns grupos de maior risco, o que elevou os gastos epidemiológicos, mas o controle da doença é gradual e ainda vem sendo realizado. Apesar desse fato ter ocorrido quatro anos antes do período inicial de análise desse estudo, deve-se ponderar que as ações e decisões tomadas em áreas como a saúde podem demorar a surtir efeito, ainda mais ao se considerar que as políticas de prevenção dessa doença iniciaram após a instauração do surto no país.

Dadas as particularidades sobre a Aids e a Gripe Influenza, parte-se para a análise dos casos de Dengue e dos focos do mosquito transmissor. Ainda de acordo com a Tabela 5, verifica-se que esses modelos não são significativos, assim como suas variáveis explicativas. Portanto, não há confiança estatística, dentro dos parâmetros estabelecidos, para realizar qualquer inferência de impacto dos gastos em vigilância epidemiológica nos casos de Dengue e nos focos do Aedes aegypti. Diante disso, realizou-se alguns testes na busca por modelos mais adequados, com as seguintes alterações: (i) utilização do logaritmo natural para diminuição das escalas; e (ii) exclusão de outliers, por meio da técnica de amplitude do interquartil, acrescido de uma constante. Os resultados das quatro regressões (casos e focos, com logaritmo natural e casos e focos sem outliers) mostraram-se significativos, mas quando analisadas as variáveis explicativas de forma isolada, em nenhum dos modelos os gastos com vigilância epidemiológica apresentaram significância, o que reitera a limitação de inferências dessa relação.

Por fim, cabe destacar que a população, além de correlacionar-se com todas as doenças (Tabela 4), possui impacto comprovado na Gripe Influenza e na Aids (Tabela 5); ou seja, quanto maior a população, maior a ocorrência dessas enfermidades. Isso corrobora os achados de Santos et al. (2002), Preaud et al. (2014) e Araújo et al. (2017), de que as doenças epidemiológicas se propagam com maior facilidade nas aglomerações populacionais.

Diante dos resultados supracitados, de forma geral verifica-se que os gastos com vigilância epidemiológica explicam a ocorrência de duas das três doenças analisadas, porém em sentido inverso ao esperado pela literatura relativa ao tema. Assim, como análise complementar, para compreender melhor essas relações, estima-se um novo modelo de regressão, em que o gasto passa a ser a variável dependente e os casos de doenças as variáveis explicativas, cujos resultados se apresentam na Tabela 6. 
Tabela 6

Resultado da regressão - determinantes dos gastos em vigilância epidemiológica

\begin{tabular}{lccc}
\hline $\mathrm{N}^{\mathbf{0}}$ de observações & 240 & $\mathrm{~F}(6,59)$ & 2,16 \\
$\mathrm{R}$-Squared & 0,3651 & Prob > F & 0,0595 \\
\hline \multicolumn{1}{c}{ Gripe Influenza } & $\mathbf{t}$ & $\mathbf{P}>\mathbf{t}$ & Coeficiente \\
Dengue casos & 2,27 & 0,027 & $7.388,494$ \\
Dengue focos & $-3,34$ & 0,001 & $-274,7933$ \\
Aids & $-1,51$ & 0,136 & $-203,1288$ \\
População & 2,08 & 0,042 & $5.190,191$ \\
Área & $-0,26$ & 0,797 & $-0,4762369$ \\
cons & $-1,17$ & 0,246 & $-64,88276$ \\
\hline
\end{tabular}

Fonte: Elaborada pelos autores com base nos dados da pesquisa (2019).

De acordo com a Tabela 6, o modelo é significativo e as variáveis dependentes explicam, conjuntamente, $36,51 \%$ dos gastos com vigilância epidemiológica. Ao analisar as variáveis individualmente, as três enfermidades (Gripe Influenza, Dengue e Aids) possuem significância estatística, sendo que a Gripe e a Aids elevam os gastos dessa subfunção (cada caso registrado aumenta os gastos em $\mathrm{R} \$ 7.388,49$ e $\mathrm{R} \$ 5.190,19$, respectivamente), enquanto os casos de Dengue diminuem o seu valor ( $-\mathrm{R} \$ 274,79$ por caso). Os focos do mosquito transmissor, bem como as duas variáveis de controle, não apresentam significância estatística, o que limita as inferências.

Ao analisar os resultados de todas as regressões (Tabelas 5 e 6 ), de forma geral tem-se um cenário em que maiores gastos com vigilância epidemiológica implicam, também, mais casos de doenças. Por sua vez, mais casos de doenças aumentam os gastos. Isso pode significar que os recursos públicos destinados a essa subfunção apresentam uma conotação de tratamento em vez de prevenção, o que diverge das recomendações, uma vez que, segundo a Dive (2017), esses recursos deveriam direcionar-se a ações para o conhecimento, a detecção e a prevenção de doenças. Contudo, deve-se ponderar que o ônus econômico de uma epidemia, quando esta é instaurada, é expressivo, pois requer ações emergenciais e com impacto nos diferentes setores da economia (Dih-Ling et al., 2018). Assim, por mais que a incidência de ações para tratamento seja menor, as diferenças monetárias em relação às ações preventivas podem influenciar tais resultados.

\section{CONCLUSÃO}

Das três doenças analisadas no presente estudo, apenas a Dengue (casos e focos do mosquito) não permite inferências quanto ao impacto de gastos em vigilância epidemiológica na mitigação de seus casos. Para as demais, ao contrário do esperado pela literatura (Liu \& Mills, 2002, Bein et al., 2017, Lins et al., 2019) e pelo apelo populacional, pode-se verificar que quanto maior os gastos nessa subfunção, mais casos dessas doenças são evidenciados.

Contudo, não é possível aferir que os gastos em vigilância epidemiológica são, necessariamente, ineficientes na mitigação dessas doenças, uma vez que podem apresentar apenas um direcionamento contrário ao esperado; não atuando de forma preventiva, mas curativa, com foco no tratamento. Diante disso, em análise complementar, pode-se constatar que quanto mais casos de Gripe e Aids, maiores os gastos alocados a essa subfunção, o que reitera que as escolhas públicas podem apresentar desvios do propósito central do Sistema Único de Saúde, que é a prevenção. O fato de a análise dos focos de Dengue não apresentar significância em nenhum modelo pode contribuir para essa afirmação, já que, dentre as variáveis analisadas, esta é a única integralmente destinada à prevenção de doenças. Além disso, deve-se ponderar que as ações em saúde podem não surtir efeitos imediatos e os recursos alocados em determinado período tornamse eficientes para a mitigação de doenças apenas em períodos posteriores.

Por mais que este estudo não permita inferências sobre as reais motivações para a tomada de decisão dos gestores públicos, no sentido de verificar se agem por interesse próprio - ou não - 
conforme preceitos da TEP (Buchanan \& Tullock, 1962), as discussões aqui apresentadas demonstram que existe um comportamento que difere do esperado e que, portanto, necessita de uma melhor compreensão para garantir a eficiência na aplicação dos recursos públicos. Este estudo também aponta outros fatores dificultadores do planejamento em saúde e que podem influenciar as decisões dos gestores, como os decorrentes de atrasos na alimentação das informações no sistema (Santos et al., 2002) e as distorções quanto ao local de incidência dos casos, devido à procura pelos grandes centros de referência em saúde. Além disso, McKibbin e Roshen (2020, p. 26) alegam que muitos políticos "continuam ignorando as evidências científicas sobre o papel da saúde pública na melhoria da qualidade de vida e como propulsora do crescimento econômico". Portanto, sugere-se que estudos futuros explorem de forma mais detalhada e extensiva as causas desse comportamento, já que os preceitos da TEP podem não ser suficientes para explicar a tomada de decisão diferente da expectativa dos eleitores.

Cabe destacar que os dados aqui pesquisados referentes ao período de 2013 a 2016, de acordo com Liu e Mills (2002) e Bein et al. (2017), são suficientes para refletir o impacto no número de casos de Aids, Dengue e Gripe Influenza, uma vez que alguns gastos públicos geram melhorias imediatas para a saúde da população. Mas como os resultados estatísticos não confirmaram tal relação, talvez seja necessário coletar dados temporais mais longos, como sugerido por Halici-Tülüce et al. (2016), com mais de um mandato dos prefeitos; o que pode possibilitar, também, uma análise com mais especificidade para as diferentes escolhas públicas, bem como reduzir os problemas estatísticos de autocorrelação e não normalidade dos resíduos.

A necessidade de pesquisas mais profundas com foco em áreas especificas da saúde, como o realizado neste estudo para vigilância epidemiológica, é iminente. A maioria dos estudos, em especial os internacionais, tende a utilizar a classificação contábil padrão da OCDE, que insere em uma conta sintética todos os gastos com saúde. Contudo, a utilização de dados em nível macro e sua generalização para o nível micro pode ser pouco realista, haja vista que cada governo e cada região tem suas especificidades, além da saúde ser uma área altamente especializada com características distintas. Diante disso, é necessário o redirecionamento das contas públicas, sendo que o primeiro passo é entender como os gastos se comportam.

\section{REFERÊNCIAS}

Albuquerque, A. C. de, Cesse, E. Â. P., Felisberto, E, Samico, I. C., \& Frias, P. G. de. (2019). Avaliação de desempenho da regionalização da vigilância em saúde em seis Regiões de Saúde brasileiras. Cadernos de Saúde Pública, 35(2), 1-16. doi:10.1590/0102-311x00065218

Araújo, V. E. M., Bezerra, J. M. T., Amâncio, F. F., Passos, V. M. A., \& Carneiro, M. (2017). Aumento da carga de dengue no Brasil e unidades federadas, 2000 e 2015: análise do Global Burden of Disease Study 2015. Revista Brasileira de Epidemiologia, 20(1), 205-216. doi: 10.1590/1980-5497201700050017

Barbosa, L. G. (2013). Análise dos gastos em saúde com as vigilâncias do campo da saúde nos municípios de Minas Gerais: 2005 a 2011. Dissertação de Mestrado, Programa de Mestrado em Saúde Pública, Escola Nacional de Saúde Pública - Fundação Oswaldo Cruz, Rio de Janeiro, RJ, Brasil.

Barreto, M. L., \& Souza, L. E. (2015). Saúde coletiva precisa de pesquisa e inovação. Abrasco, 1, 1-18. ISBN: 978-85-85740-05-4

Bein, M. A., Unlucan, D., Olowu, G., \& Kalifa, W. (2017). Healthcare spending and heal thoutcomes: evidence from selected East African countries. African Health Sciences, 17(1), 247-254. doi: 10.4314/ahs.v17i1.30 
Bousmaha, M. Q., Ventelou, B., \& Abu-Zaineh, M. (2016). Medicine and democracy: The importance of institutional quality in the relationship between health expenditure and health outcomes in the MENA region. Health Policy, 120(8), 928-935. doi: 10.1016/j.healthpol.2016.06.005

Buchanan, J. M., \& Tullock, G. (1962). The Calculus of Consent: Logical Foundations of Constitutional Democracy. Ann Arbor. MI: University of Michigan Press.

Constituição da República Federativa do Brasil [CRFB] (1988). Recuperado de http://www.planalto.gov.br/ccivil_03/Constituicao/Constituicao.htm

Costa, G. P. C. L., \& Gartner, I. R. (2017). O efeito da função orçamentária alocativa na redução da desigualdade de renda no Brasil: uma análise dos gastos em educação e saúde no período de 1995 a 2012. Revista de Administração Pública - RAP, 51(2), 264-293. doi: 10.1590/00347612155194.

Dermindo, M. P. (2019). Gestão eficiente na saúde pública brasileira. Journal of Management \& Primary Health Care, 11. doi:10.14295/jmphc.v11iSup.933

Dih-Ling, L., Cheng-Chieh, L., Yun-Ru, L., \& Szu-Chieh, C. (2018). Economic cost and burden of dengue during epidemics and non-epidemic years in Taiwan. Journal of Infection and Public Health, 11(2), 215-223.

Diretoria de Vigilância Epidemiológica [Dive] (2006). O perfil epidemiológico da AIDS. Santa Catarina: Brasil. Recuperado de: http://www.dive.sc.gov.br/conteudos/publicacoes/livros_artigos/O_perfil_epidemiologico_da _AIDS.pdf

Diretoria de Vigilância Epidemiológica [Dive] (2017). Doenças e Agravos. Santa Catarina: Brasil. Recuperado de: http://www.dive.sc.gov.br/index.php/doencas-agravos

Ensor, T., Chhun, C., Kimsun, T., McPake B., \& Edoka I. (2017). Impact of health financing policies in Cambodia: 20 year experience. Social Science \& Medicine, 177, 118-126. doi: 10.1016/j.socscimed.2017.01.034

Fabre, V. V., Scheeffer, F., Dallabona, L. F., \& Kroetz, M. (2018). Analysis of expenditures with environmental management on the political-ideological behavior of managers, from the point of view of the theory of political choices. Brazilian Journal of Development, 4(2), 514-531.

Gujarati, D. (2006). Econometria básica (4a ed.). Trad. Maria José Cyhlar Monteiro, Rio de Janeiro: Elsevier.

Halici-Tülüce, N. S., Dogan, I., \& Dumrul, C. (2016). Is income relevant for health expenditure and economic growth nexus? International Journal of Health Economics and Management, 16(1), 23-49. doi: 10.1007/s10754-015-9179-8

Holmes, S., \& Sunstein, C. R. (2000). The cost of rights: why liberty depends on taxes. New York: Norton. 
Lapsley, I., \& Wright, E. (2004). The diffusion of management accounting innovations in the public sector: a research agenda. Management Accounting Research, 15, 355-374.

Lei n. 8.080, de 19 de setembro de 1990. (1990). Dispõe sobre as condições para a promoção, proteção e recuperação da saúde, a organização e o funcionamento dos serviços correspondentes e dá outras providências. Recuperado de http://www.planalto.gov.br/ccivil_03/Leis/L8080.htm

Lins, J. G. M. G, Ciríaco, J. S., \& Anjos, O. R., (2019). Avaliando o impacto do financiamento federal no controle epidemiológico da dengue no Brasil. Revista Brasileira de Economia de Empresas, 19(2), 23-38.

Liu, X., \& Mills, A. (2002). Financing reforms of public health services in China: lessons for other nations. Social Science \& Medicine, 54, 1691-1698.

McKibbin, W. J. \& Roshen, F. (2020). The Global Macroeconomic Impacts of COVID-19: Seven Scenarios. CAMA, Centre for Applied Macroeconomic Analysis, Working Paper, 19. doi: $10.2139 /$ ssrn.3547729

Mladenovi, I., Milovancevi, M., Mladenovi, S. S., Marjanovi V., \& Petkovi, B. (2016). Analyzing and management of healthcare expenditure and gross domestic product (GDP) growth rate by adaptive neuro-fuzzy technique. Computers in Human Behavior, 64, 524-530. doi: 10.1016/j.chb.2016.07.052

Morais, L. M. D., Vicente, E. F. R., \& Souza, G. P. (2016). Efeitos da Lei de Responsabilidade Fiscal sobre a tendência de despesas públicas do estado de Santa Catarina: evidências no período 1995-2010 segundo critérios da OCDE1. Revista da CGU, 8(12), 311-350.

Naseem, A., Rashid, A., \& Kureshic, N. I. (2014). E-health: effect on health system efficiency of Pakistan. Ann Saudi Med, 34(1), 59-64. doi: 10.5144/0256-4947.2014.59

Pan, J., \& Liu, G. G. (2012). The determinants of Chinense Provincial Government health expenditure: evidence from 2002-2006 data. Health Economics, 21, 757-777. doi: 10.1002/hec. 1742

Preaud, E., Durand, L., Macabeo, B., Farkas, N., Sloesen, B., Palache, A., Shupo, F., \& Samson, S. I. (2014). Annual public health and economic benefits of seasonal influenza vaccination: European estimate. BMC Public Health, 14(1), 813-824. doi:10.1186/1471-2458-14-13

Santos, N. J. S., Tayra, A., Silva, S. R., Buchalla, C. M., \& Laurenti, R. (2002). As mudanças no perfil da epidemia e perspectivas da vigilância epidemiológica. Revista Brasileira de Epidemiologia, 5(3), 286-310.

Secretaria do Tesouro Nacional [STN]. (2017). Manual de Contabilidade Aplicado ao Setor Público. (7a ed.), Brasília: Brasil.

Temporão, J. G. (2009). O enfrentamento do Brasil diante do risco de uma pandemia de influenza pelo vírus A (H1N1). Epidemiologia e Serviços de Saúde, 18(3), 201-204. 
Worrall, E., Rietveld, A., \& Delacollette, C. (2004). The burden of malária epidemic and costeffectiveness of intervention in epidemic situation in Africa. The American Journal of Tropical Medicine and Hygiene, 71(2), 136-140.

Younsi, M., Chakroun, M., \& Nafluma, A. (2016). Robust analysis of the determinants of healthcare expenditure growth: evidence from panel data for low-, middle- and high-income countries. The International journal of health planning and management, 31(4), 580-601. doi: 10.1002/hpm.2358

Zhu, Q., \& Wang, X. (2011). Structural changes of public expenditures in China. Journal of Public Budgeting - Accounting \& Financial Management, 23(4), 569-587. 